\title{
Origin of the Short-Range, Strong Repulsive Force between Ionic Surfactant Layers
}

\author{
Jordi Faraudo* \\ Departament de Fisica, Universitat Autonoma de Barcelona, Facultat de Ciencies, E-08193 Bellaterra, Spain
}

Fernando Bresme ${ }^{\dagger}$

Department of Chemistry, Imperial College London, Exhibition Road, London, SW7 2AZ, United Kingdom

(Received 29 June 2004; published 23 February 2005)

\begin{abstract}
We study the electrostatic interaction between two ionic surfactant layers by performing molecular dynamic simulations of salt-free thin water films coated by surfactants (Newton black films). We find a strong exponentially decaying short-range repulsion not explained by classical Poisson-Boltzmann theory. This electrostatic force is shown to be mainly due to the anomalous dielectric response of water near charged surfactant layers. This result clarifies the much debated physical mechanism underlying the controversial "hydration forces" observed in experiments. In the case of ionic thin films, the "hydration forces" can be identified with the electrostatic forces induced by the layers of highly polarized water originated at the interfaces.
\end{abstract}

DOI: 10.1103/PhysRevLett.94.077802

Introduction. - The Derjaguin-Landau-Verwey-Overbeek (DLVO) theory [1-3] provides a successful explanation of the long range interaction forces observed in a great variety of systems (colloids, surfactant and lipid bilayers, etc.), in terms of electrostatic and van der Waals forces. However, the DLVO theory is inaccurate in the case of surfaces separated by small distances. The deviations between experiments and DLVO predictions are usually interpreted by invoking the existence of short-range forces different from electrostatic and van der Waals forces [4,5]. It is fair to say that the physical origin of many of these non-DLVO forces is still obscure (see [4] for a critical revision). One of the most widely studied and controversial non-DLVO force is the so-called "hydration force" [6], a strong short-range repulsive force that decays exponentially with the distance, $H$, between the surfaces:

$$
\Pi=\Pi_{0} e^{-H / \lambda} .
$$

The concept of "hydration force" emerged to explain measurements of forces between neutral lipid bilayer membranes [6]. Its presence in charged systems is controversial, but there is experimental evidence of non-DLVO forces following Eq. (1) in systems as diverse as dihexadecyldimethyl ammonium acetate surfactant bilayers $\left(\Pi_{0} \approx 10^{9}-10^{10} \mathrm{~Pa}, \lambda \approx 3.0 \AA\right.$ ) [7], DNA polyelectrolytes [8] (with $\lambda \approx 2.5-3.5 \AA$ ), and charged polysaccharide [9] (with $\Pi_{0}=7.3 \times 10^{9} \mathrm{~Pa}, \lambda \approx 3.3 \AA$ ) [6]. In these experiments, the "hydration forces" show very weak sensibility to ionic strength.

The physical mechanisms underlying the hydration force are still open to debate. One possible mechanism is the anomalous polarization of water near the interfaces, which completely alters its dielectric response [10-13]. In these theories, the hydration force has an electrostatic origin. However, other authors claim [14] that there is no evidence for a significant structuration of water layers near
PACS numbers: 68.15.+e, 02.70.Ns, 68.05.Cf, 82.45.Mp

interfaces, or a perturbation of its dielectric response, as assumed by previous theories. Instead, they propose that the repulsive forces are due to entropic (osmotic) repulsion of thermally excited molecular groups that protrude from the surfaces [14-16]. This theory explains many experimental observations in neutral systems [15], but it is not clear its validity in charged systems. In our opinion, with the present evidence from experiments and simulations, it is not possible to give a definitive answer on the precise role of these mechanisms in determining the hydration forces. In fact, our computer simulations of water films coated with ionic surfactants show that protrusions are not significant in these systems [17]. On the other hand, our simulations show that water has an anomalous dielectric behavior near charged interfaces [18], but the observed electrostatic fields clearly differ from the predictions of electrostatic theories on hydration forces [11,12]. The effect that this anomalous dielectric behavior of water has on the electrostatic force between surfaces or interfaces is still unknown.

Our aim in this Letter is to study the electrostatic interaction between ionic surfactant layers taking into account the anomalous dielectric behavior of water [18]. At short distances we observe a strong electrostatic repulsion, which is due to the anomalous dielectric response of water near the surfactant layers. This electrostatic short-range repulsion can be easily identified with the so-called "hydration force."

Simulation details. - We study the interaction between ionic surfactant layers considering thin water films of different sizes sandwiched between two layers of sodium dodecylsulfate (SDS) surfactants $\left[\mathrm{CH}_{3}\left(\mathrm{CH}_{2}\right)_{11} \mathrm{OSO}_{3}{ }^{-} \mathrm{Na}^{+}\right]$. This surfactant is widely employed in science and technology, and thin films consisting of SDS/water/SDS have been investigated experimentally [19]. The detailed description of the force field for the surfactant as well as all other simulation details can be 
found in Ref. [20]. Our simulations were performed at constant temperature $T=298 \mathrm{~K}$. The surface area per surfactant was $A / N_{\mathrm{SDS}}=33 \AA^{2}$, corresponding to the value found in experiments [19]. We have considered films from $H \approx 32 \AA$ [where $H$ is the size of the aqueous core defined as the mean distance between the $\mathrm{O}$ (ester) atoms in surfactants located in different monolayers] down to contact between surfactant layers $(H \approx 6 \AA)$. Films of different sizes are obtained by considering simulations with different number $N_{w}$ of extended simple point charge (SPC/E) water molecules [21]. The observed change of $H$ with $N_{w}$ corresponds to that expected for a film filled with liquid water with density $\approx 1 \mathrm{~g} / \mathrm{cm}^{3}$. We have shown [20] that the predictions of this computational model are in very good agreement with the available structural experimental data (number and electron density, roughness, correlation functions, etc.). Since the model has been validated, we employ it in the following to investigate electrostatic interactions in thin films.

In a top view of the films (see the auxiliary material [22]), one can clearly observe clusters with $\mathrm{DS}^{-}$and $\mathrm{Na}^{+}$ ions in the $(x, y)$ plane. To calculate the interactions between the surfactant layers, one needs to know whether these structures are affected by decreasing the film thickness $H$. To address this point we have computed the radial distribution functions $g(\rho)$, where $\rho=\sqrt{x^{2}+y^{2}}$ is the projection on the $x, y$ plane of the distance between two particles. Figure 1 shows the function $g_{\mathrm{SNa}}(\rho)$ characterizing the transversal correlations between head groups and $\mathrm{Na}^{+}$ions for films with different $H$. The structure of the ion cloud in the $x, y$ plane [as characterized by $g_{\mathrm{SNa}}(\rho)$ ] practically remains unchanged as $H$ decreases. The same conclusion can be obtained analyzing the distribution functions $g_{\mathrm{SS}}(\rho)$ and $g_{\mathrm{NaNa}}(\rho)$ for different $H$ values. These results show that the distribution of $\mathrm{DS}^{-}$and $\mathrm{Na}^{+}$ions in the plane $x, y$ and, consequently, the electrostatic fields generated by them, do not depend on the film thickness. This remarkable result is consistent with theoretical studies of electrostatic interactions using approximations beyond the mean field theory. Calculations of the effect of discrete surface charges on the electrostatics of thin aqueous films [23] showed that the ion distribution in the $x, y$ direction is independent of the film thickness. Our results and those in Ref. [23] correspond to monovalent ions. For multivalent ions, these correlations could be very different [24].

From now on, we average all physical quantities obtained from simulations (ion distribution, electric fields, etc.) over the $x, y$ plane. This procedure is fully justified because we have shown that the structure of the film in these directions $(x, y)$ is essentially independent of the film thickness $H$. Our procedure differs from the one used in a mean field (MF) approach. In the latter the fields and densities are obtained from equations that neglect a priori their dependence with the parallel direction $x, y$. The Poisson-Boltzmann (PB) equation and all present theories on hydration forces $[11,12]$ belong to this class of MF theories. In contrast, we have computed the exact distribution of charges for the simulation model, and the physical quantities of interest are then averaged a posteriori. An example of the dramatic difference of neglecting the $x, y$ dependence a priori and a posteriori for the case of colloidal interactions is given in Ref. [25].

Approximate calculation of electrostatic interactions. Our simulations of Newton black films show that water exhibits an anomalous, nonlocal, dielectric response [18]. A dielectric permittivity cannot be defined inside the films because there is no local relation between the electric field $\vec{E}$ and the polarization of water $\vec{P}$. The absence of a local relation between $\vec{E}$ and $\vec{P}$ has been predicted in Refs. [11,12]. However, the electrostatic potential and fields predicted by these theories do not agree with our simulations [18]. The theoretical approaches introduce certain hypotheses on the mechanisms governing the structure of water near the interfaces. We consider instead the exact fields and particle distributions from the computer simulations. These are then used to compute the electrostatic Helmholtz free energy $F_{\mathrm{el}}$ of the films.

The electrostatic free energy of the film is usually split into two contributions [2,3], $F(H)=F_{\mathrm{fd}}+F_{\text {mix }} . F_{\mathrm{fd}}$ is due to the presence of an electric field inside a medium, and $F_{\text {mix }}$ accounts for the inhomogeneous mixing of the $\mathrm{Na}^{+}$ions due to the electric field. First, we compute the free energy of the field $F_{\mathrm{fd}}(H)$ using (see Chap. 3 in [26])

$$
F_{\mathrm{fd}}(H)=F_{\mathrm{fd}}^{(0)}+\frac{A}{2} \int \rho_{f}(z) \phi(z) d z
$$

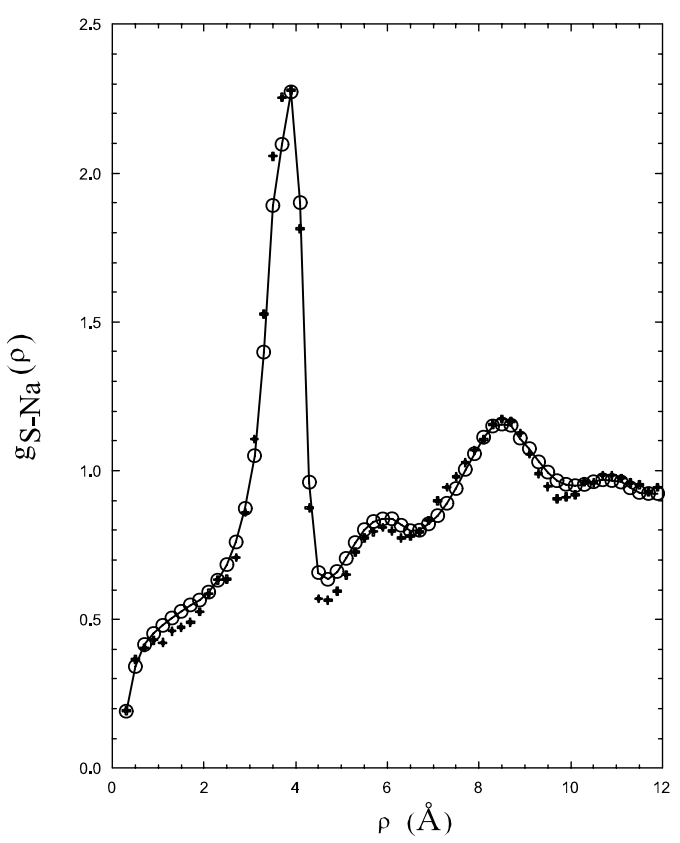

FIG. 1. Correlation function $g_{\mathrm{SNa}}(\rho)$ showing the structure of the ion cloud in the $x, y$ plane for film thicknesses $H \approx 24.8 \AA$ (circles connected by a line) and $H \approx 8.7 \AA$ (crosses). 
where $\phi(z)$ is the electrostatic potential, $\rho_{f}(z)$ is the socalled density of free charges defined as the charge density computed excluding the charges pertaining to the molecules of the dielectric medium, i.e., water. $F_{\text {fd }}^{(0)}$ is an arbitrary origin for the free energy. We set $F_{\mathrm{el}}=0$ for the largest film. $F_{\mathrm{fd}}(H)-F_{\mathrm{fd}}^{(0)}$ is the reversible work involved in the process of extraction of the "free" charges from its actual positions to infinite separation. In Fig. 2 we show the result obtained from Eq. (2) [the product $\rho_{f}(z) \phi(z)$ was averaged over equilibrium configurations, typically over a time interval of 1-1.5 ns and the integration was computed numerically]. For comparison, we also show the predictions of the PB equation. Surprisingly, $F_{\mathrm{fd}}$ originates a repulsive contribution to the interaction between the surfactant layers. In the classical PB theory, the field free energy provides a strong attractive contribution to the interaction (the overall electrostatic repulsion predicted by $\mathrm{PB}$ is due to the large repulsive contribution of the $F_{\text {mix }}$ term). The differences between the fields observed in our system and those predicted by PB theory are due to the anomalous dielectric response of water. The exact contribution of this effect to the free energy [Eq. (2)] can be determined as follows. We construct an imaginary system in which we replace the observed distribution of water molecules by a continuum medium with the dielectric constant of water $\epsilon_{r}\left(\epsilon_{r} \approx 70\right.$ for the SPC/E model of water [27] at $T=298 \mathrm{~K}$ ). We consider for the $\mathrm{DS}^{-}$and $\mathrm{Na}^{+}$ions the charge distributions obtained in the simulations. In this imaginary system, the electric displacement $\vec{D}$ is the same as that obtained in the simulations and the electric field is now given by $\vec{E}=\vec{D} / \varepsilon_{r} \varepsilon_{0}$. Hence, in the imaginary system the field free energy given by Eq. (2) has to be replaced by

$$
F_{\mathrm{fd}}^{*}(H)=F_{\mathrm{fd}}^{(0)}+\frac{A}{2 \varepsilon_{r} \varepsilon_{0}} \int \vec{D}(z) \cdot \vec{D}(z) d z .
$$

The difference between $F_{\mathrm{fd}}$ and $F_{\mathrm{fd}}^{*}$ accounts for the effect of the anomalous dielectric response. The comparison between $F_{\mathrm{fd}}$ and $F_{\mathrm{fd}}^{*}$ shown in Fig. 2 demonstrates that the main contribution to the short-range electrostatic repulsion is due to the perturbation of water induced at the interfaces.

Now, let us analyze the results for the total electrostatic free energy $F_{\text {el }}=F_{\text {fd }}+F_{\text {mix }}$. The mixing term $F_{\text {mix }}$ is computed using the classical expression:

$$
\begin{aligned}
F_{\text {mix }}= & A k_{B} T \int n_{\mathrm{Na}}(z) \ln \frac{n_{\mathrm{Na}}(z)}{n_{\mathrm{Na}}(z)+n_{w}(z)} d z \\
& +A k_{B} T \int n_{w}(z) \ln \frac{n_{w}(z)}{n_{\mathrm{Na}}(z)+n_{w}(z)} d z,
\end{aligned}
$$

where $n_{\mathrm{Na}}(z)$ and $n_{w}(z)$ are, respectively, the number density of $\mathrm{Na}^{+}$ions and water molecules. $F_{\mathrm{el}}(H)$ as computed from our simulations and Eqs. (4) and (2) is shown in Fig. 3. For comparison, we also show $F_{\mathrm{el}}(H)$ calculated

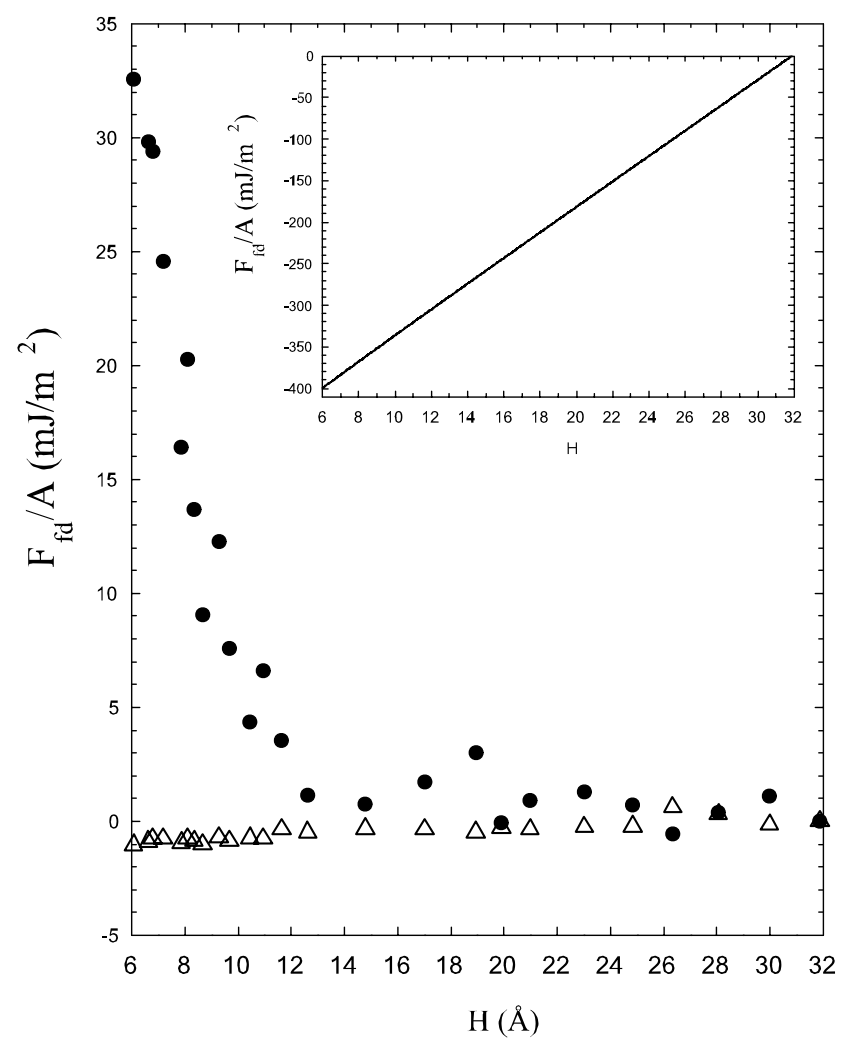

FIG. 2. Field contribution to the electrostatic free energy of the film. Filled circles correspond to $F_{\mathrm{fd}}$ computed in simulations using Eq. (2), and triangles correspond to $F_{\mathrm{fd}}^{*}$ computed assuming that water behaves as an ordinary dielectric [Eq. (3)]. Inset: prediction of the PB theory, assuming that approximately $10 \%$ of the SDS surfactants are ionized $[18,20]$.

using the fields and ion distribution predicted by the PB equation. The simulation results show that at short distances between the surfactant layers the free energy is strongly repulsive. This electrostatic repulsion is mainly due to the structuring of water near the surfactant layers. The repulsion has the typical exponential behavior of the hydration force. The electrostatic force per unit surface $\Pi_{\mathrm{el}}$ between the surfactant layers is given by [2] $\Pi_{\mathrm{el}}=-\frac{1}{A} \times$ $\left.\frac{d F_{\text {el }}}{d H}\right|_{T}$. Combining this equation with Eq. (1) one has $F=$ $F_{0}+\Pi_{0} \lambda A e^{-H / \lambda}$. A fit of our simulation data to this expression gives $\lambda=2.35 \AA$ and $\Pi_{0}=4.09 \times 10^{9} \mathrm{~Pa}$, in clear agreement with typical experimental values [6]. A clear evidence for the existence of a repulsive force in Newton black films is the stability of freestanding SDS/ water/SDS films $(H=7.5 \AA)$ obtained in [19]. In these films, a repulsive force opposing the attractive van der Waals force must exist. Using our values for $\lambda$ and $\Pi_{0}$ in Eq. (1), we obtain a repulsive force of $\Pi=$ $1.7 \times 10^{8} \mathrm{~Pa}$. The experimental value of the Hamaker constant for these films is $A_{\mathrm{H}}=(4-30) \times 10^{-20} \mathrm{~J}$ [28] and the distance between van der Waals planes is approximately $D \approx 3.7 \AA$; therefore, the van der Waals attractive 


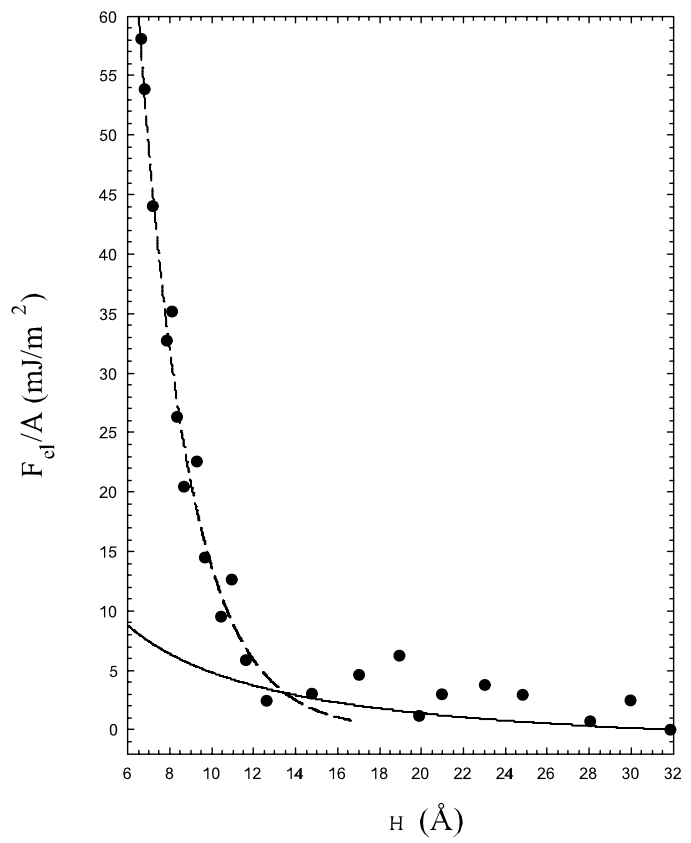

FIG. 3. Electrostatic free energy of the Newton black film. Full circles correspond to $F_{\text {el }}$ computed in simulations [Eqs. (2) and (4)]. The dashed line is an exponential fit to $F_{\text {el }}$ in the range $H<$ $12 \AA$. The fitting parameters are given in the text. The solid line is the prediction of the $\mathrm{PB}$ theory, obtained considering that $\approx 10 \%$ of the SDS surfactants are dissociated $[18,20]$.

pressure is $\quad \Pi=A_{\mathrm{H}} /\left(6 \pi D^{3}\right) \approx(0.4-3.1) \times 10^{8} \mathrm{~Pa}$. According to this, the repulsive force predicted here is of the same order of magnitude than the attractive van der Waals force, and therefore it should play an important role in determining the film stability.

Conclusions. - We have shown that the electrostatic interactions between ionic surfactant layers exhibit a strongly repulsive short-range behavior that is directly related to the anomalous dielectric response of water near interfaces. This nonclassical electrostatic repulsive force is qualitatively and quantitatively similar to the socalled hydration force observed in a variety of charged systems. We believe that our results may help to clarify the much debated physical origin of the hydration force.

This work has been supported by EPSRC Research Grant No. GR/R39726/01. Simulations were performed at the HPCx supercomputing center (Edinburgh, U.K.) under the Materials Chemistry consortium (U.K.). J.F. also acknowledges financial support from the Spanish Government under Grant No. BFM2000-0351-C03-01.

*Electronic address: Jordi.Faraudo@uab.es
†Electronic address: f.bresme@imperial.ac.uk

[1] J. Israelachvili, Intermolecular and Surface Forces (Academic Press, New York, 1991).

[2] D. F. Evans and H. Wennerström, The Colloidal Domain (Wiley-VCH, New York, 1999).

[3] E. J.W Verwey and J.Th. G. Overbeek, Theory of the Stability of Lyophobic Colloids (Elsevier, New York, 1948).

[4] B. W. Ninham, Adv. Colloid Interface Sci. 83, 1 (1999).

[5] V. Bergeron, J. Phys. Condens. Matter 11, R215 (1999).

[6] S. Leikin, V. A. Parsegian, and D.C. Rau, Annu. Rev. Phys. Chem. 44, 369 (1993).

[7] V. A. Parsegian, R. P. Rand, and N.L. Fuller, J. Phys. Chem. 95, 4777 (1991).

[8] D..C. Rau, B. K. Lee, and V.A. Parsegian, Proc. Natl. Acad. Sci. U.S.A. 81, 2621 (1984).

[9] D.C. Rau and V. A. Parsegian, Science 249, 1278 (1990).

[10] S. Marcelja, Nature (London) 385, 689 (1997).

[11] D. W. R. Gruen and S. Marcelja, J. Chem. Soc., Faraday Trans. 2 79, 225 (1983); S. Marcelja and N. Radic, Chem. Phys. Lett. 42, 129 (1976).

[12] E. Ruckenstein and M. Manciu, Langmuir 18, 7584 (2002); M. Manciu and E. Ruckenstein, Adv. Colloid Interface Sci. 112, 109 (2004).

[13] S. Leikin and A. A. Kornishev, J. Chem. Phys. 92, 6890 (1990).

[14] J. Israelachvili and H. Wennerstrom, Nature (London) 379, 219 (1996).

[15] J. N. Israelachvili and H. Wennerstrom, Langmuir 6, 873 (1990); J. N. Israelachvili and H. Wennerström, J. Phys. Chem. 96, 520 (1992).

[16] L. Leckband and J. N. Israelachvili, Q. Rev. Biophys. 34, 105 (2001).

[17] F. Bresme and J. Faraudo (unpublished).

[18] J. Faraudo and F. Bresme, Phys. Rev. Lett. 92, 236102 (2004).

[19] O. Bélorgey and J. J. Benattar, Phys. Rev. Lett. 66, 313 (1991).

[20] F. Bresme and J. Faraudo, Langmuir 20, 5127 (2004).

[21] H. J. C. Berendsen, J. R. Grigera, and T. P. Straatsma, J. Phys. Chem. 91, 6269 (1987).

[22] See EPAPS Document No. E-PRLTAO-94-017510 for snapshots of simulations of two different films. A direct link to this document may be found in the online article's HTML reference section. The document may also be reached via the EPAPS homepage (http://www.aip.org/ pubserves/epaps.html) or from ftp.aip.org in the directory /epaps/. See the EPAPS homepage for more information.

[23] L. Foret, R. Kuhn, and A. Wurger, Phys. Rev. Lett. 89, 156102 (2002).

[24] J. C. Butler et al., Phys. Rev. Lett. 91, 028301 (2003).

[25] J. Faraudo and J. Bafaluy, Europhys. Lett. 46, 505 (1999).

[26] P. Lorrain and D. Corson, Electromagnetic Fields and Waves (W. H. Freeman, New York, 1970).

[27] P. Höchtl et al., J. Chem. Phys. 109, 4927 (1998).

[28] K. P. Velikov et al., Faraday Transactions 93, 2072 (1997). 